\title{
The Development and Use of Online Tools in Microscopy and Microanalysis Facilities.
}

Miles Apperley ${ }^{1}$, Jenny Whiting ${ }^{1}$, Bronwen $\mathrm{Cribb}^{2}$, Christopher Frost ${ }^{2}$, Anna Ceguerra ${ }^{3}$, Peter Liddicoat $^{3}$ and Carlos Aya ${ }^{4}$

1. Australian Microscopy and Microanalysis Research Facility, Madsen Building F09, The University of Sydney, NSW, 2006, Australia.

2. Center for Microscopy and Microanalysis, The University of Queensland, Brisbane, QLD, 4072, Australia

3. Australian Center for Microscopy and Microanalysis and the School of Aerospace, Mechanical and Mechatronic Engineering, Madsen Building F09, The University of Sydney, NSW, 2006, Australia.

4. Intersect Australia Ltd., Sydney, NSW 2000 Australia

The Australian Microscopy and Microanalysis Research Facility (AMMRF) is a national grid of equipment, instrumentation and expertise in microscopy and microanalysis that provides nanostructural characterisation capability and services, from widely used optical, electron, X-ray and ion-beam techniques to world-leading flagship platforms. The latter include pulsed-laser local-electrode atom probe, high-throughput cryo-transmission electron microscopy, high-resolution scanning electron microscopy and spectroscopy, as well as high-precision ion microprobe and secondary-ion mass spectrometers. This collaborative facility, comprising a distributed network of microscopy and microanalysis core facilities spread over fourteen institutions, manages a fully supported user experience to more than 3,000 researchers annually.

The typical research experience of a user commences with the stages of defining the scientific question and project formulation, identifying techniques and registering the project at an appropriate facility. This is followed by a meeting to discuss the project, plan training and arrange access to instrumentation. The later stages include data acquisition and analysis, data management and ultimately an outcome such as a research publication, grant application or invention registration or patent. With more than 3,000 users annually there is a strong need to adopt processes that enable users to be productive as well as to enable efficient management by facility staff at all stages of the user experience.

One of the benefits of the network of core facilities is the ability to collaborate to develop online tools that are then accessible by all the laboratories in the network. In addition to web-based instrument booking systems, and other resource management tools, that are widely used by many core facilities, the AMMRF has developed specialist tools for microscopy and microanalysis research facilities. These tools assist researchers to identify the techniques they need to use, facilitate training and enable data analysis \& management.

The Technique Finder (TF) is a web application that enables prospective facility users to identify the techniques most suited to their research, based on a researcher-centric approach and terminology as opposed to instrument-oriented jargon. Specifically, it offers two areas, one for biological scientists and another for researchers in physical sciences, which allow them to identify techniques based on research dimensions in corresponding fields. In addition, it offers a term search based on a comprehensive term index created for each technique including all the directly and indirectly linked information available in the application. The techniques themselves display a full description with sample examples, key reviews 
and potential for case studies and links. Locations and contact details to each of the AMMRF facilities invite users to get started immediately.

MyScope: Training for Advanced Research, is an online suite of education tools for teaching and learning in the area of microscopy and microanalysis. A range of modules has been developed to sit within the suite: scanning electron microscopy; transmission electron microscopy; scanning probe and atomic force microscopy; confocal microscopy; and X-ray diffraction techniques. The modules in MyScope contain a number of components including: an interactive questionnaire to allow the user to assess their knowledge, guide choices and tailor the learning environment for flexible learning; self guided tutorials with videos, animations and glossary to prepare students with knowledge and specialist language; virtual instrument platforms to practice use of instrumentation; and online competency testing to demonstrate readiness for hands-on experience.

A Data Management System (DMS) addresses the needs of an increasing number of AMMRF users who are using high-end instruments to produce large datasets. Those users are facing the demands of a new wave of data-intensive instruments and software that enable: higher spatial resolution; higher chemical resolution; 3D and 4D+ approaches; more rapid dynamic processes; and multi-dimensional analyses. To make effective use of these capabilities, researchers must have easy access to a wide range of sophisticated data management, processing and analysis tools. The DMS offers a uniform user interface to access remote or local data and metadata resources, effectively leveraging the web as Software as a Service platform for data management. It provides basic protocol interfaces, allowing AMMRF users to leverage common storage infrastructure as well as the potential to connect to a range of online analysis tools or platforms.

A specific analysis platform being developed is the Atom Probe Workbench. This tool is a component of a larger national eResearch project in Australia, that is aiming to integrate existing tools and techniques with a network of specialised cloud-based computing systems and data-storage facilities. This integration will enable the atom probe research community to access and create valuable tools, accelerating the research process. Features of the workbench will include the atom probe DMS system that performs automatic ingestion of data \& meta-data directly from instruments, processing-modules for accessing reconstruction and analysis techniques, and a visualisation engine for exploring data and preparation of publication-quality images [1].

\section{References:}

[1] The authors acknowledge support from the following Commonwealth funding programs: National Collaborative Research Infrastructure Strategy; National eResearch Architecture Taskforce; Office of Learning and Teaching; and National eResearch Collaboration Tools and Resources (NeCTAR) project. 